Research Article

\title{
Iterative Data-Driven Control for Closed Loop with Two Unknown Controllers
}

\author{
Hong Jianwang $\left(\mathbb{D}\right.$, Ricardo A. Ramirez-Mendoza ${ }^{\mathbb{D}}$, and Ruben Morales-Menendez $\mathbb{i}$ \\ School of Engineering and Sciences, Tecnologico de Monterrey, Monterrey, Mexico \\ Correspondence should be addressed to Hong Jianwang; 9120180002@jxust.edu.cn
}

Received 12 June 2021; Revised 8 July 2021; Accepted 27 July 2021; Published 4 August 2021

Academic Editor: Zain Anwar Ali

Copyright (c) 2021 Hong Jianwang et al. This is an open access article distributed under the Creative Commons Attribution License, which permits unrestricted use, distribution, and reproduction in any medium, provided the original work is properly cited.

\begin{abstract}
Iterative idea is combined with data-driven control and is used to design the feedforward controller and feedback controller simultaneously. Consider one closed loop system with two controllers, the classical model-based control holds on the condition of known plant. To alleviate the modeling process for plant, data-driven control is applied to design the two controllers. After these two controllers are parametrized by two unknown parameter vectors, the iterative idea is introduced to identify these two parameter vectors. Furthermore, for more general case of controllers, the closed relations between controllers and expected transfer functions are derived. Then, the iterative idea is also introduced to achieve the controller design. To be of benefit for latter stability analysis, some equities are derived for output-input sensitivity functions with three kinds of disturbances. Generally, after formulating the problem of the controller design as one model-matching problem, the purpose of this paper is threefold. First, we derive that, in case of two parametrized controllers, the iterative idea is performed to identify these two unknown parameter vectors, even when parameters converge to their true values. Second, we show how to design the two controllers iteratively for more general forms and find the closed relations between these controllers and expected closed loop transfer functions. Third, we provide some heuristic considerations on output-input sensitivity functions, which are of benefit for our stability analysis on datadriven control. Finally, one example is given to show the feasibility of our proposed theories.
\end{abstract}

\section{Introduction}

Data-driven control is one new advanced control strategy in theory and practice, due to its lack of system modeling process. Classical control strategy, i.e., model-based control, depends on the mathematical model greatly of the considered system or plant. More specifically, when to control one plant in the open loop or closed loop, the first step is to construct one mathematical model for this plant by using first principle or system identification; then, this obtained mathematical model is applied for the latter controller design or other missions. During the last 60 years, the main task of system identification concerns on finding one approximated model to best fit the observed input-output data. Based on this approximated model, a suitable controller is designed under the condition that this identified model converges to its real system. Generally, model-based control includes two steps: system identification and controller design. In recent years, researchers are thinking about how to combine these two steps, i.e., designing controller directly from the observed input-output data. Then, data-driven control is generated to achieve the controller design from data without the modeling process. Especially during our information age, lots of observed data are very easy to yield, so it is necessary to study data-driven control and apply it in some real engineering fields, such as paper production, glass production, and separation process like crystallization. To alleviate the dependence on the identified model for the controller, the notion of the data-driven approach is widely studied in recent years. The attracting property of the datadriven approach is that the controller is designed directly based on measured data. As the data-driven approach is still in its infancy, different names are called in the references to describe it, such as data-driven, data-based, and model-free. 
To the best of our knowledge, the principles between datadriven approach and system identification are similar to each other, as the measured data are applied to get the mathematical model for the unknown plant in the framework of system identification, but get the approximated controller for the case of the data-driven approach. The idea of direct data-driven control was first proposed in machine learning; then, it attracted many researchers in the advanced control field recently. Now, this data-driven theory is widely applied in the control field, for example, direct data-driven control, data-driven estimation, datadriven detection, and data-driven optimization. The common property among them is that the measured data are used to achieve our main goals; then, it means some useful information are extracted from these measured data. On the contrary, the data-driven approach needs lots of measured data, i.e., the number of measured data is sufficiently large. This requirement is feasible in our information period, and the data-driven approach was born to overcome the limitation of the model-based approach, so the data-driven approach is studied very popularly from theory and practice application.

Due to no model identification process for data-driven control, the unknown controllers, whatever feedback controller and feedforward controller, are yielded through the observed input-output data. We say that lots of information exist in observed data, for example, information for system modeling, information for controller design, and information for fault detection. The first is named as system identification, which is studied during these 40 years, but the second corresponds to data-driven control, proposed in these 10 years, so the research on system identification is very mature, such as identification algorithm and convergence analysis. In these 10 years, many detailed strategies for data-driven control are proposed to generate the controller from observed data, for example, virtual reference feedback tuning control, subspace predictive control, iterative correction tuning control, and set membership predictive control. All the above data-driven control strategies hold on the condition that the observed data must be persistent exciting or data informative, and it means all the implicit modes in system and controllers are excited perfectly; then, their complete properties are included in the observed data. The task of data-driven control is to apply some ways to extract these useful properties for the system (system identification) and controller (data-driven control). By the way, the third information for fault detection corresponds to testify the faults from the observed data, specifically in UAVs' formation control and modern war. For example, in recent years, we have succeeded in applying ellipsoidal approximation in target tracking for multi-UAVs' formation cooperative detection. Its essence is to identify the unknown state from the observed data, as some important factors are in the state estimation.

The idea of data driven can be combined with other control strategies, such as adaptive control, internal model control, robust control, and model predictive control. It is similar to a new concept-identification for control, proposed in 2010s, to combine system identification and other control strategies. Consider the case of data-driven model predictive control, system identification is used to identify one function estimation; then, this obtained function estimation is substituted into the cost function for model predictive control. As the number of references on data-driven control is vast, we cannot list all of them here, so some are given to be suitable for our own study. Data-driven control is applied to minimize the energy consumption in [1], where the mathematical equation for the energy consumption is constructed as one linear state space system. Formulas for data-driven control are analyzed, such as stabilization, optimality, and robustness in [2], where the detailed linear feedback controller is expressed as one matrix form about the data Hankel matrix. The closed relations between modelbased control and data-driven control are studied to point out the perspective for data-driven control [3]. Consider one nonlinear feedback loop system [4], data-driven control is introduced to design controllers with two degrees of freedoms. And, set membership identification is more widely studied for the case of unknown but bounded noise. Campi and Weyer [5] proposed one new data-driven control strategy, i.e., virtual reference feedback tuning control; furthermore, finite sample properties of it are derived for the finite sample data points. Then, this sample complexity is considered for the classical linear quadratic regulator [6]; as a consequence, this sample complexity is the required number of data points which makes the control performance to achieve the desired one. Last year, some researchers started to derive data informativity for data-driven analysis and control [7], from the point of persistency excitation [8]. One important condition on optimal input must be satisfied to guarantee the Hankel matrix be full rank. As reinforcement learning is very popular now, so this reinforcement learning will be also introduced in control theory [9]; it tells reinforcement learning will be a hot subject for data-driven control in future. During these 3 years, we also publish some contributions on data-driven control, for example, zonotope parameter identification for data-driven control [10] and finite sample properties for data-driven control [11]. The idea about combining data-driven control with adaptive control is seen in [12], where Lyapunov stability is applied to derive one parameter adjustment law, satisfying the stability for the whole adaptive system. Research on piecewise affine system identification are still done, for example, Bianchi and Breschi [13] developed a general framework to alternate between parameter estimation and sample mode assignment. In reference [14], given a set of input-output data, the identification of a switched nonlinear system for the underlying system involves the simultaneous identification of the mode sequence. Due to the application of the data-driven approach widely in the control field and the similar point between data-driven approach and system identification, we call their combination as identification for control, i.e., system identification for direct data-driven control. More specifically, we describe a concise introduction or contribution on system identification for direct data-driven control, which belongs to the data-driven approach. In case of the unknown but bounded noise, one bounded error identification is proposed to identify the unknown systems 
with time varying parameters. Then, one feasible parameter set is constructed to include the unknown parameter with a given probability level. The problem about how to construct this confidence interval is solved by a linear approximation/ programming approach [15], which can identify the unknown parameter only for the linear regression model.

Based on our previous contributions on data-driven control [10-12], this paper introduces the iterative idea into data-driven control, as the iterative idea exists in theory and practice more widely. Roughly speaking, the parameter converges to its true values as an iterative form until the estimation error be zero. Consider one closed loop system with two degrees of freedom controllers, i.e., feedback controller and feedforward controller, data-driven control strategy is used to design these two unknown controllers from the observed input-output data. When given the expected closed loop transfer function and expected sensitivity function, the problem of designing these two unknown controllers is reduced to one model-matching problem, whose optimization variables correspond to the two unknown controllers. Moreover, one cost function is constructed based on the observed input-output data for this model-matching problem. After formulating the problem of the controller design as one model-matching problem, the purpose of this paper is threefold. First, we derive that, in case of two parametrized controllers, the iterative idea is performed to identify these two unknown parameter vectors, even when parameters converge to their true values. Second, we show how to design the two controllers iteratively for more general forms and find the closed relations between these controllers and expected closed loop transfer functions. Third, we provide some heuristic considerations on output-input sensitivity functions, which are of benefit for our stability analysis on datadriven control.

This paper is organized as follows. In Section 2, the structure of the considered closed loop system with two unknown controllers i.e., feedback controller and feedforward controller, is formulated. For the sake of completeness, the classical model-based control is reviewed and one short introduction on data-driven control is also recalled from [10]. When the two unknown controllers are parametrized by two unknown parameter vectors, respectively, the iterative idea is proposed to identify these two unknown parameter vectors in Section 3, which means parameterized controllers design iteratively. Then, for more general forms on these two unknown controllers, the detailed relations between them and expected closed loop transfer function are established in Section 4; then, the iterative idea is applied again. In Section 5, more kinds of noise and disturbances are considered in this same closed loop system. Then, some derivations about output-input sensitivity functions are given for our latter stability analysis for data-driven control. In Section 6, one example shows the effectiveness of the proposed iterative controller design. Section 7 ends the paper with final conclusion and points out the next interesting subject. Here, in this paper, all the mathematical derivations are obtained by our own contributions.

\section{Preliminaries}

We start by recalling the results given in $[10,11]$.

2.1. Closed Loop System with Two Controllers. Consider one closed loop system with two controllers in Figure 1.

In Figure 1, $r(t)$ is the external input signal, $P(z)$ is the unknown plant, and it can be denoted as one proper rational transfer function form. $C_{1}(z)$ and $C_{2}(z)$ are two unknown controllers, i.e., feedforward controller and feedback controller, respectively. The main task of this paper is to design these two unknown controllers. $u(t)$ and $y(t)$ are the input and output for the considered plant $P(z) . d(y)$ is one kind of external disturbance, which will be extended in Section 5 . $\varepsilon(t)=r(t)-C_{2}(z) y(t)$ is the error signal, imposed on that feedforward controller $C_{1}(z)$. During the framework of data-driven control, two unknown controllers $\left\{C_{1}(z), C_{2}(z)\right\}$ are designed based on the observed inputoutput data $\{u(t), y(t)\}$, while avoiding the system identification process for that unknown plant $P(z) . z$ is one time shift operator, i.e., $z u(t)=u(t-1)$.

Observing Figure 1 again, two controllers exist in that closed loop system simultaneously, i.e., the common unit feedback is replaced by feedback controller $C_{2}(z)$. The benefit of these two controllers is that they cooperate with each other to achieve the control mission. More specifically, the considered closed loop with two controllers appears in some practical engineers, such as flight control, servo control, and predictive control.

Consider that, in Figure 1, we have the following relations such as

$$
\begin{aligned}
y(t 1) & =P(z) u(t)+d(t) \\
& =P(z) C_{1}(t) \mathcal{E}(t)+d(t), \\
\mathcal{E}(t) & =r(t)-C_{2}(t) y(t) .
\end{aligned}
$$

From above equation (1), the complete input-output equation for $\{r(t), y(t)\}$ is that

$$
\begin{aligned}
y(t)= & \frac{P(z) C_{1}(z)}{1+P(z) C_{1}(z) C_{2}(z)} r(t) \\
& +\frac{1}{1+P(z) C_{1}(z) C_{2}(z)} d(t) .
\end{aligned}
$$

Set the transfer function from $r(t)$ to $y(t)$ be the closed loop transfer function, and similarly, the other transfer function from $d(t)$ to $y(t)$ is the sensitivity function. For the sake of completeness, the classical model-based control is formulated as follows, given the expected closed loop transfer function and expected sensitivity function as $M(z)$ and $S(z)$; then, the problem of the controller design is to design the two controllers $\left\{C_{1}(z), C_{2}(z)\right\}$ so as to make the closed loop transfer function and sensitivity function be equal to their own expected forms $M(z)$ and $S(z)$. Generally, the classical model-based control is formulated as the following model-reference problem: 


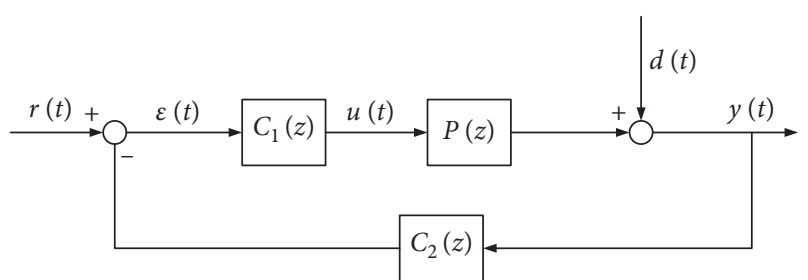

Figure 1: Closed loop system with two controllers.

$$
\begin{aligned}
\min _{C_{1}(z), C_{2}(z)} J_{1}(\theta, \eta)= & \left\|\frac{P(z) C_{1}(z)}{1+P(z) C_{1}(z) C_{2}(z)}-M(z)\right\|_{2}^{2} \\
& +\left\|\frac{1}{1+P(z) C_{1}(z) C_{2}(z)}-S(z)\right\|_{2}^{2}
\end{aligned}
$$

where $\|\cdot\|_{2}^{2}$ is Euclidean norm and $\left\{C_{1}(z), C_{2}(z)\right\}$ are two optimization variables.

From [10], model reference problem (3) is solvable on the condition that plant $P(z)$ is known, so firstly the model identification process is needed to give one mathematical equation for that unknown plant $P(z)$. To avoid this model identification process for plant $P(z)$, data-driven control is applied.
2.2. Data-Driven Control. Based on the above description on classical model-based control, the reason about why that plant $P(z)$ exists in cost function (3) is that the input-output data $\{r(t), y(t)\}$ are used for the whole closed loop system, not the input-output data $\{u(t), y(t)\}$ with respect to that unknown plant $P(z)$. To be more precise, if the input-output data $\{u(t), y(t)\}$ around plant $P(z)$ are observed, some information about two unknown controllers $\left\{C_{1}(z), C_{2}(z)\right\}$ are included in these input-output data $\{u(t), y(t)\}$, not $\{r(t), y(t)\}$.

Consider that given closed loop transfer function $M(z)$, the relation between input $u(t)$ and output $y(t)$ is that

$$
\begin{aligned}
u(t) & =C_{1}(z, \theta) \varepsilon(t) \\
& =C_{1}(z, \theta)\left(M^{-1}(z)-C_{2}(z, \eta)\right) y(t) .
\end{aligned}
$$

Similarly, consider that given sensitivity function $S(z)$, it also holds that

$$
u(t)=-C_{1}(z, \theta) C_{2}(z, \eta) \frac{S(z)}{S(z)-1} y(t) .
$$

Equations (4) and (5) are the relations for input $u(t)$ and output $y(t)$, corresponding to the given closed loop transfer function $M(z)$ and given sensitivity function $S(z)$. Combining equations (4) and (5) simultaneously, two unknown controllers $\left\{C_{1}(z), C_{2}(z)\right\}$ are designed through the following optimization problem:

$$
\begin{aligned}
\min _{C_{1}(z), C_{2}(z)} J_{2}\left(C_{1}(z), C_{2}(z)\right)= & \frac{1}{N} \sum_{t=1}^{N}\left[u(t)-C_{1}(z)\left(M^{-1}(z)-C_{2}(z)\right) y(t)\right]^{2} \\
& +\frac{1}{N} \sum_{t=1}^{N}\left[u(t)+C_{1}(z) C_{2}(z) \frac{S(z)}{S(z)-1} y(t)\right]^{2},
\end{aligned}
$$

where $M^{-1}(z)$ and $S^{-1}(z)$ are two inverse transfer functions and $N$ is the number of observed data. From [10], that unknown pant $P(z)$ does not exist in cost function (6), i.e., no modeling process for plant $P(z)$. Input-output data $\{u(t), y(t)\}_{t=1}^{N}$ are observed, and $\{M(z), S(z)\}$ are given, so only two controllers $\left\{C_{1}(z), C_{2}(z)\right\}$ are optimization variables in cost function (6). This is the essence for our considered data-driven control. All above detailed introduction on closed loop system and data-driven control are seen in our previous contributions $[10,11]$.

\section{Parametrized Controller Design}

Parametrized controller is always common in practice, such as the PID controller. The merit of the parametrized controller is to change the formal controller design to one problem of parameter estimation. It means after the parameter is identified, then the controller is obtained through the simple substitution.

For convenience, two controllers $\left\{C_{1}(z), C_{2}(z)\right\}$ are parametrized by two unknown parameter vectors $\theta$ and $\eta$, respectively, i.e., $\left(C_{1}(z, \theta), C_{2}(z, \eta)\right)$. As a consequence, the parametrized forms are listed as follows:

$$
\begin{aligned}
C_{1}(z, \theta) & =\alpha^{T}(z) \theta, \\
C_{2}(z, \eta) & =\beta^{T}(z) \eta, \\
\alpha(z) & =\left[\alpha_{1}(z), \alpha_{2}(z), \ldots, \alpha_{n}(z)\right]^{T}, \\
\beta(z) & =\left[\beta_{1}(z), \beta_{2}(z), \ldots, \beta_{n}(z)\right]^{T}, \\
\theta & =\left[\theta_{1}, \theta_{2}, \ldots, \theta_{n}\right]^{T}, \\
\eta & =\left[\eta_{1}, \eta_{2}, \ldots, \eta_{n}\right]^{T},
\end{aligned}
$$

where in equation (7), $\alpha(z)$ and $\beta(z)$ are two known orthogonal basic functions and $\{\theta, \eta\}$ are tow unknown parameter vectors with suitable dimension $n$. After two unknown controllers $\left\{C_{1}(z), C_{2}(z)\right\}$ are parametrized by two unknown parameter vectors $\{\theta, \eta\}$, i.e., $\left\{C_{1}(z), C_{2}(z)\right\}$, then corresponding optimization problem (6) is rewritten as that 


$$
\begin{aligned}
\min _{\theta, \eta} J_{3}(\theta, \eta)= & \frac{1}{N} \sum_{t=1}^{N}\left[u(t)-C_{1}(z, \theta)\left(M^{-1}(z)-C_{2}(z, \eta)\right) y(t)\right]^{2} \\
& +\frac{1}{N} \sum_{t=1}^{N}\left[u(t)+C_{1}(z, \theta) C_{2}(z, \eta) \frac{S(z)}{S(z)-1} y(t)\right]^{2}
\end{aligned}
$$

And, the parametrized closed loop system is replotted in Figure 2.

Observing optimization problems (6) and (7), the controller design is reduced to identify those unknown parameter vectors $\{\theta, \eta\}$. As the pointwise infimum of a family of affine function of $\{\theta, \eta\}$ is concave, so those two terms in equation (7) are convex function. Moreover, the first term is rewritten as

$$
\begin{gathered}
u(t)-C_{1}(z, \theta)\left(M^{-1}(z)-C_{2}(z, \eta)\right) y(t)=u(t) \\
-\alpha^{T}(z) \theta M^{-1}(z) y(t)-\alpha^{T}(z) \theta \beta^{T}(z) \eta y(t) .
\end{gathered}
$$

It corresponds to one geometric programming; then, use the variables defined as $x=\log \theta$ and $y=\log \eta$, so $\theta=e^{x}$ and $\eta=e^{y}$, and it can be changed as one simple linear optimization problem. There are lots of optimization methods for solving equation (7), such as least squares method, first order gradient method, and set membership method. Whatever optimization methods are used, the iterative idea is introduced to identify the two unknown parameter vectors $\{\theta, \eta\}$. Generally, the complete parameter estimation process with the iterative idea is formulated as follows.

Step 1: given two initial parameter vectors $\left\{\theta_{0}, \eta_{0}\right\}$.

Step 2: compute the first parameter vector through solving the following optimization problem, and denote it as $\widehat{\theta}_{1}$ :

$$
\begin{aligned}
\widehat{\theta}_{1}= & \underset{\theta}{\arg \min } J_{3}\left(\theta, \eta_{0}\right) \\
= & \frac{1}{N} \sum_{t=1}^{N}\left[u(t)-C_{1}(z, \theta)\left[M^{-1}(z)-C_{2}\left(z, \eta_{0}\right)\right] y(t)\right]^{2} \\
& +\frac{1}{N} \sum_{t=1}^{N}\left[u(t)+C_{1}(z, \theta) C_{2}\left(z, \eta_{0}\right) \frac{S(z)}{S(z)-1} y(t)\right]^{2} .
\end{aligned}
$$

Step 3: similarly, compute the second parameter vector through solving one optimization problem, and denote it as $\widehat{\eta}_{1}$ :

$$
\begin{aligned}
\widehat{\eta}_{1}= & \underset{\eta}{\arg \min } J_{3}\left(\theta_{0}, \eta\right) \\
= & \frac{1}{N} \sum_{t=1}^{N}\left[u(t)-C_{1}(z, \theta)\left(M^{-1}(z)-C_{2}(z, \eta)\right) y(t)\right]^{2} \\
& +\frac{1}{N} \sum_{t=1}^{N}\left[u(t)+C_{1}(z, \theta) C_{2}(z, \eta) \frac{S(z)}{S(z)-1} y(t)\right]^{2} .
\end{aligned}
$$

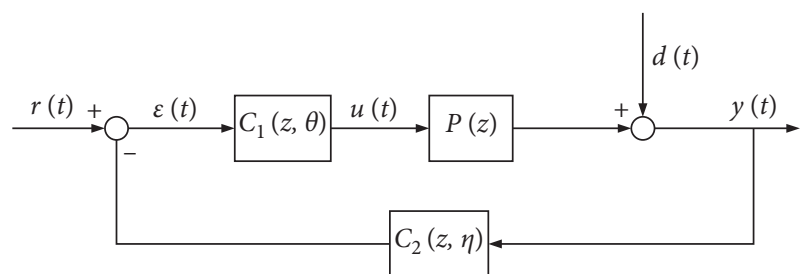

Figure 2: Closed loop system with two parametrized controllers.

Step 4: iterate to generate parameter estimation $\widehat{\theta}_{2}$ :

$$
\begin{aligned}
\widehat{\theta}_{2}= & \underset{\theta}{\arg \min } J_{3}\left(\theta, \widehat{\eta}_{1}\right) \\
= & \frac{1}{N} \sum_{t=1}^{N}\left[u(t)-C_{1}(z, \theta)\left(M^{-1}(z)-C_{2}\left(z, \widehat{\eta}_{1}\right)\right) y(t)\right]^{2} \\
& +\frac{1}{N} \sum_{t=1}^{N}\left[u(t)+C_{1}(z, \theta) C_{2}\left(z, \hat{\eta}_{1}\right) \frac{S(z)}{S(z)-1} y(t)\right]^{2} .
\end{aligned}
$$

Step 5: iterate to generate parameter estimation $\widehat{\eta}_{2}$ :

$$
\begin{aligned}
\widehat{\eta}_{2}= & \underset{\eta}{\arg \min } J_{3}\left(\hat{\theta}_{1}, \eta\right) \\
= & \frac{1}{N} \sum_{t=1}^{N}\left[u(t)-C_{1}\left(z, \widehat{\theta}_{1}\right)\left(M^{-1}(z)-C_{2}(z, \eta)\right) y(t)\right]^{2} \\
& +\frac{1}{N} \sum_{t=1}^{N}\left[u(t)+C_{1}\left(z, \widehat{\theta}_{1}\right) C_{2}(z, \eta) \frac{S(z)}{S(z)-1} y(t)\right]^{2} .
\end{aligned}
$$

Step $i$ : based on the obtained parameter estimations $\left\{\widehat{\theta}_{i-1}, \widehat{\eta}_{i-1}\right\}$, the new parameter estimations $\left\{\widehat{\theta}_{i}, \widehat{\eta}_{i}\right\}$ are computed iteratively:

$$
\begin{aligned}
& \widehat{\theta}_{i}=\underset{\theta}{\arg \min } J_{3}\left(\theta, \widehat{\eta}_{i-1}\right), \\
& \widehat{\eta}_{i}=\underset{\eta}{\arg \min } J_{3}\left(\widehat{\theta}_{i-1}, \eta\right) .
\end{aligned}
$$

When the following inequality holds, then terminate the above iterative process:

$$
\left\|\widehat{\theta}_{i}-\widehat{\theta}_{i-1}\right\|_{2}+\left\|\widehat{\eta}_{i}-\widehat{\eta}_{i-1}\right\|_{2} \leq \varepsilon
$$

where $\epsilon$ is one positive value with sufficient small, for example, $\varepsilon=0.1$.

Equation (14) embodies our iterative idea, and it means when to compute the $i$ th parameter estimation $\widehat{\theta}_{i}$, the other parameter estimation $\eta$ in the cost function is substituted by its $i-1$ th parameter estimation $\widehat{\eta}_{i-1}$. And, it is the same process for computing $\widehat{\eta}_{i}$. 


\section{Iterative Controller Design}

The parametrized controllers $\left(C_{1}(z, \theta), C_{2}(z, \eta)\right)$ are considered in Section 3, where the problem of the controller design is changed to the parameter estimation. As those two unknown parameter vectors $\{\theta, \eta\}$ exist in cost function (7) as the multiply form, so our proposed iterative idea is introduced to identify them separately. During all of our previous contributions, we do not concern on the closed relations between the close loop transfer functions and their expected forms $\{M(z), S(z)\}$. Here, our tasks are to find these closed relations and apply them into design more general controllers $\left(C_{1}(z), C_{2}(z)\right)$ iteratively.

Combining equations (4) and (5), two controllers $\left(C_{1}(z), C_{2}(z)\right)$ must satisfy that

$C_{1}(z)\left(M^{-1}(z)-C_{2}(z)\right) y(t)=-C_{1}(z) C_{2}(z) \frac{S(z)}{S(z)-1} y(t)$.

It means that

$$
\begin{aligned}
M^{-1}(z)-C_{2}(z) & =-C_{2}(z) \frac{S(z)}{S(z)-1}, \\
(S(z)-1) M^{-1}(z)-(S(z)-1) C_{2}(z) & =-C_{2}(z) S(z), \\
(S(z)-1) M^{-1}(z)+C_{2}(z) & =0 .
\end{aligned}
$$

Then, feedback controller $C_{2}(z)$ is yielded as follows:

$$
C_{2}(z)=\frac{1-S(z)}{M(z)} \text {. }
$$

Equation (18) shows after we have the expected closed loop transfer function $M(z)$ and expected sensitivity function $S(z)$, then feedback controller $C_{2}(z)$ is easily obtained from equation (18), so the next question is to get the other feedforward controller $C_{1}(z)$. After simple but tedious calculations, we have the following equities:

$$
\begin{aligned}
C_{2}(z) M(z) & =\frac{P(z) C_{1}(z) C_{2}(z)}{1+P(z) C_{1}(z) C_{2}(z)}, \\
1-C_{2}(z) M(z) & =1-\frac{P(z) C_{1}(z) C_{2}(z)}{1+P(z) C_{1}(z) C_{2}(z)} \\
& =\frac{1}{1+P(z) C_{1}(z) C_{2}(z)}, \\
\frac{\left[1-C_{2}(z) M(z)\right] P(z)}{M(z)}= & \frac{P(z)}{1+P(z) C_{1}(z) C_{2}(z)} \\
& \times \frac{1+P(z) C_{1}(z) C_{2}(z)}{P(z) C_{1}(z)}, \\
= & \frac{1}{C_{1}(z)} .
\end{aligned}
$$

Then, the feedforward controller $C_{1}(z)$ is yielded as that

$$
C_{1}(z)=\frac{M(z)}{\left[1-C_{2}(z) M(z)\right] P(z)},
$$

and substituting equation (18) into (20), then we have

$$
C_{1}(z)=\frac{M(z)}{\left[1-C_{2}(z) M(z)\right] P(z)}=\frac{M(z)}{S(z) P(z)} .
$$

Observing equations (18) and (21), when $M(z)$ and $S(z)$ are known in advance, then feedback controller $C_{2}(z)$ is obtained easily, but plant $P(z)$ is needed to derive the feedforward controller $C_{1}(z)$. As a consequence, feedback controller $C_{2}(z)$ can be generated from these two expected transfer functions $\{M(z), S(z)\}$, but information of plant $P(z)$ is needed in feedforward controller $C_{1}(z)$. Here, the problem about information $P(z)$ and designing $C_{1}(z)$ can be handled by the iterative idea. Generally, the complete controller design process for more general controllers is formulated as follows:

Step 1: given two expected or design transfer functions $\{M(z), S(z)\}$

Step 2: compute the feedback controller $C_{2}(z)$ directly by

$$
C_{2}(z)=\frac{1-S(z)}{M(z)}
$$

Step 3: choose one initial feedforward controller $C_{1}^{0}(z)$ as

$$
C_{1}^{0}(z)=\frac{M(z)}{S(z) P_{0}(z)}
$$

where $P_{0}(z)$ is the initial plant

Step 4: using $C_{1}^{0}(z)$ and fixed $C_{2}(z)$, determine plant $P_{1}(z)$ as

$$
P_{1}(z)=\operatorname{argmin}_{P(z)}\left\|\frac{P(z) C_{1}^{0}(z)}{1+P(z) C_{1}^{0}(z) C_{2}(z)}-M(z)\right\|_{2}^{2} .
$$

Step 5: substitute $P_{1}(z)$ into $C_{1}(z)$ to give one new feedforward controller $C_{1}^{1}(z)$, i.e.,

$$
C_{1}^{1}(z)=\frac{M(z)}{S(z) P_{1}(z)}
$$

Step $i$ : iterate to compute the $i$ th plant $P_{i}(z)$ and the $i$ th feedforward controller $C_{1}^{i}(z)$ :

$$
\begin{aligned}
& P_{i}(z)=\operatorname{argmin}_{P(z)}\left\|\frac{P(z) C_{1}^{i-1}(z)}{1+P(z) C_{1}^{i-1}(z) C_{2}(z)}-M(z)\right\|_{2}^{2}, \\
& C_{1}^{i}(z)=\frac{M(z)}{S(z) P_{i}(z)} .
\end{aligned}
$$


When the following inequity holds, then terminate the above iterative process:

$$
\left\|P_{i}(z)-P_{i}(z)\right\|_{2}+\left\|C_{1}^{i}(z)-C_{1}^{i-1}(z)\right\|_{2} \leq \varepsilon .
$$

Comment: the above iterative process solves the identification for plant $P(z)$ and feedforward controller $C_{1}(z)$, as feedback controller $C_{2}(z)$ is known from the given transfer functions $\{M(z), S(z)\}$. Then, the the iterative process is related with plant $P(z)$ and feedforward controller $C_{1}(z)$ within the framework of data driven control.

\section{Design Analysis}

The above theoretical analysis about the iterative design for data-driven control concerns on the closed loop system in Figure 1. As only output disturbance $d(t)$ exists in Figure 1, so it is one ideal case. But on the contrary in practice, maybe three kinds of disturbances appear in the considered closed loop system in Figure 3, i.e., output disturbance, input disturbance, and measurement noise.

In Figure $3, d(t)$ denotes output disturbance, $d_{1}(t)$ denotes the input disturbance, and $d_{2}(t)$ is measurement noise.

Similarly, for Figure 3, some relations hold based on the linear system theory. For notational clarity, we abbreviate the parameter vectors $\{\theta, \eta\}$ in following mathematical derivations:

$$
\begin{aligned}
y(t) & =d(t)+P(z) u(t) \\
& =d(t)+P(z)\left[d_{1}(t)+C_{1}(z)\left[r(t)-C_{2}(z)\left(y(t)+d_{2}(t)\right)\right]\right],
\end{aligned}
$$

i.e.,

$$
\begin{gathered}
{\left[1+P(z) C_{1}(z) C_{2}(z)\right] y(t)=d(t)+P(z) d_{1}(t)} \\
+P(z) C_{1}(z) r(t)-P(z) C_{1}(z) C_{2}(z) d_{2}(t) .
\end{gathered}
$$

Then,

$$
\begin{aligned}
y(t)= & \frac{d(t)+P(z) d_{1}(t)+P(z) C_{1}(z) r(t)}{1+P(z) C_{1}(z) C_{2}(z)} \\
& -\frac{P(z) C_{1}(z) C_{2}(z) d_{2}(t)}{1+P(z) C_{1}(z) C_{2}(z)} .
\end{aligned}
$$

From equation (30), one transfer function from output disturbance $d(t)$ to plant output $y(t)$ is defined as follows, i.e., output sensitivity function:

$$
S_{y d}(z)=\frac{1}{1+P(z) C_{1}(z) C_{2}(z)} .
$$

Similarly, the transfer function from measurement noise disturbance $d_{2}(t)$ to plant output $y(t)$ is that noise sensitivity function:

$$
S_{y d_{2}}(z)=-\frac{P(z) C_{1}(z) C_{2}(z)}{1+P(z) C_{1}(z) C_{2}(z)} .
$$

The transfer function from input disturbance $d_{1}(t)$ to plant output $y(t)$ is that

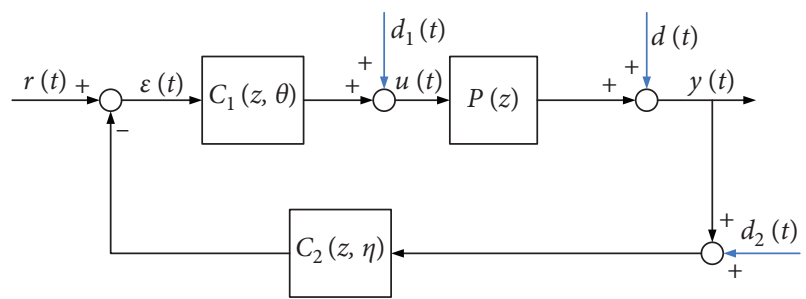

Figure 3: Extended closed loop system with three disturbances.

$$
S_{y d_{1}}(z)=-\frac{P(z)}{1+P(z) C_{1}(z) C_{2}(z)} .
$$

The transfer function from excited input $r(t)$ to plant output $y(t)$ holds that

$$
S_{y r}(z)=\frac{P(z) C_{1}(z)}{1+P(z) C_{1}(z) C_{2}(z)} .
$$

Observing equations (31) and (32), it holds that

$$
S_{y d}(z)-S_{y d_{2}}(z)=1 .
$$

If considered in the frequency domain, we have

$$
\left|S_{y d}(z)\right|+\left|S_{y d_{2}}(z)\right|=1 \text {. }
$$

Similarly, from equations (31) and (34), we have

$$
S_{y d}(z)+S_{y r}(z)=1 .
$$

Denote by $S_{1}(z)$ as the change of closed loop transfer function $S_{y r}(z)$ with the change of plant $P(z)$. Then, this $S_{1}(z)$ is derived as follows in detail:

$$
S_{1}(z)=\frac{\left(\partial S_{y r}(z) / S_{y r}(z)\right)}{(\partial P(z) / P(z))}=\frac{\partial S_{y r}(z)}{\partial P(z)} \times \frac{P(z)}{S_{y r}(z)} .
$$

From equation (34), $\left(\partial S_{y r}(z) / \partial P(z)\right)$ is computed as follows:

$$
\begin{aligned}
\frac{\partial S_{y r}(z)}{\partial P(z)}= & \frac{C_{1}(z)\left[1+P(z) C_{1}(z) C_{2}(z)\right]}{\left[1+P(z) C_{1}(z) C_{2}(z)\right]^{2}} \\
& -\frac{P(z) C_{1}(z) C_{1}(z) C_{2}(z)}{\left[1+P(z) C_{1}(z) C_{2}(z)\right]^{2}} \\
= & \frac{C_{1}(z)}{\left[1+P(z) C_{1}(z) C_{2}(z)\right]^{2}},
\end{aligned}
$$

and combining equation (38) into (39), we see that

$$
\begin{aligned}
S_{1}(z)= & \frac{\partial S_{y r}(z)}{\partial P(z)} \times \frac{P(z)}{S_{y r}(z)} \\
= & \frac{C_{1}(z)}{\left[1+P(z) C_{1}(z) C_{2}(z)\right]^{2}} \times P(z) \\
& \times \frac{1+P(z) C_{1}(z) C_{2}(z)}{P(z) C_{1}(z)} \\
= & \frac{1}{1+P(z) C_{1}(z) C_{2}(z)} .
\end{aligned}
$$


From equations (40) and (32), we get

$$
\begin{aligned}
S_{1}(z)-S_{y d_{2}}(z)= & \frac{1}{1+P(z) C_{1}(z) C_{2}(z)} \\
& +\frac{P(z) C_{1}(z) C_{2}(z)}{1+P(z) C_{1}(z) C_{2}(z)} \\
= & 1 .
\end{aligned}
$$

Equation (41) tells us that closed loop transfer function $S_{y r}(z)$ will always change with plant $P(z)$, but with plant $P(z)$ changes, one equity always holds.

The above analysis corresponds to the output sensitivity function. But the input sensitivity function is extremely important in the design of the underlying linear controller used in adaptive control. More specifically, the modulus of the input sensitivity function should be low at high frequencies in order to assure a good robustness of the system with respect to additive unstructured uncertainties located in the high frequency region.

From equation (30), we have

$$
\begin{aligned}
y(t)-d(t)= & \frac{d(t)+P(z) d_{1}(t)+P(z) C_{1}(z) r(t)}{1+P(z) C_{1}(z) C_{2}(z)} \\
& -\frac{P(z) C_{1}(z) C_{2}(z) d_{2}(t)}{1+P(z) C_{1}(z) C_{2}(z)}-d(t) \\
= & \frac{P(z) d_{1}(t)}{1+P(z) C_{1}(z) C_{2}(z)}+\frac{P(z) C_{1}(z) r(t)}{1+P(z) C_{1}(z) C_{2}(z)} \\
& -\frac{P(z) C_{1}(z) C_{2}(z) d_{2}(t)}{1+P(z) C_{1}(z) C_{2}(z)}-\frac{P(z) C_{1}(z) C_{2}(z) d(t)}{1+P(z) C_{1}(z) C_{2}(z)} .
\end{aligned}
$$

Then,

$$
\begin{aligned}
u(t)= & \frac{d_{1}(t)}{1+P(z) C_{1}(z) C_{2}(z)}+\frac{C_{1}(z) r(t)}{1+P(z) C_{1}(z) C_{2}(z)} \\
& -\frac{C_{1}(z) C_{2}(z) d_{2}(t)}{1+P(z) C_{1}(z) C_{2}(z)}-\frac{C_{1}(z) C_{2}(z) d(t)}{1+P(z) C_{1}(z) C_{2}(z)} .
\end{aligned}
$$

Similarly, four input sensitivity functions are defined as

$$
\begin{gathered}
S_{u d}(z)=-\frac{C_{1}(z) C_{2}(z)}{1+P(z) C_{1}(z) C_{2}(z)}, \\
S_{u r}(z)=\frac{C_{1}(z)}{1+P(z) C_{1}(z) C_{2}(z)}, \\
S_{u d_{1}}(z)=\frac{1}{1+P(z) C_{1}(z) C_{2}(z)}, \\
S_{u d_{2}}(z)=-\frac{C_{1}(z) C_{2}(z)}{1+P(z) C_{1}(z) C_{2}(z)} .
\end{gathered}
$$

Similarly, set $S_{2}(z)$ as the change of the input sensitivity function $S_{u d}(z)$ with the change of plant $P(z)$, i.e., $S_{2}(z)$ is given that

$$
\begin{aligned}
S_{2}(z)= & \frac{\partial S_{u d}(z)}{\partial P(z)} \times \frac{P(z)}{S_{u d}(z)} \\
= & \frac{C_{1}^{2}(z) C_{2}^{2}(z)}{\left[1+P(z) C_{1}(z) C_{2}(z)\right]^{2}} \times P(z) \\
& \times \frac{1+P(z) C_{1}(z) C_{2}(z)}{C_{1}(z) C_{2}(z)} \\
= & \frac{P(z) C_{1}(z) C_{2}(z)}{1+P(z) C_{1}(z) C_{2}(z)} .
\end{aligned}
$$

Then, it holds that

$$
S_{2}(z)+S_{u d_{1}}(z)=1 .
$$

Similarly, we also have that

$$
\frac{\partial S_{u r}(z)}{\partial P(z)} \times \frac{P(z)}{S_{u r}(z)}+S_{u d_{1}}(z)=1 .
$$

Equation (46) means that the relation about the input sensitivity function $S_{u d}(z)$ changes with plant $P(z)$, and equation (47) shows the input sensitivity function $S_{u r}(z)$ varies with plant $P(z)$. Then, similarly, other equations hold for other input sensitivity functions $S_{u d_{1}}(z)$ and $S_{u d_{2}}(z)$.

\section{Simulation Example}

Now, we propose one example to illustrate the nature of the above results. Then, the data-driven control strategy is applied in controlling one brake-by-wire system whose structure is plotted in Figure 4.

The brake-by-wire (BBW) technology basically cuts the physical link between the pedal and the braking system itself. Nowadays, almost every car in the world has conventional braking systems, and the pedal is directly connected to the brakes to apply the pressure to the wheels. For BBW systems, sensors and actuators read the amount of pressure the driver applies to the pedal, and this force is transferred to all the brakes by the braking actuator. The automotive industries have been focused on improving the performance of braking systems when talking about safety and efficiency; for that, brake-by-wire systems have continuously been studied and enhanced. A BBW system is mainly composed by a pedal which sends a braking signal to an ECU where the signal is processed and some control algorithms are executed, and as an output, an electrical signal is sent to a dc motor which transmits the movement to an actuator for generating pressure over the braking pads to perform the braking action desired by the driver. A main characteristic of a BBW system is the type of actuator that is being employed; there are electrohydraulic (EHB) and electromechanical (EMB) actuators. The main difference between them is that EHB is based on an hydraulic system triggered by an electric motor or pump, while the EMB just employs an electric motor as an actuator for generating braking torque.

The closed loop control is the most common scheme for controlling systems. For this scheme, 2 controllers were tested, the SMC and the PID. Both controllers were designed 


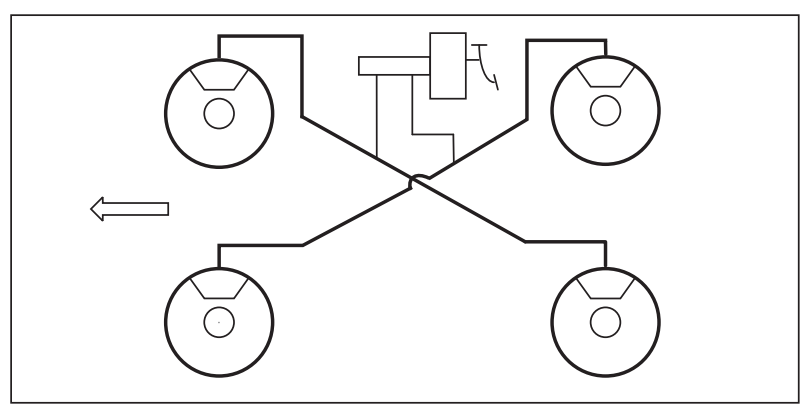

FIgURE 4: Brake-by-wire system.

based on the data-driven control. As the closed loop controllers needed to be improved in speed (from responses of hundred of milliseconds to few milliseconds), a feedforward control was proposed. The signals pressure-current in open loop was plotted and analyzed. As can be observed, the system describes a kind of exponential form with hysteresis. On the contrary, the plot generated by the error between the system with the best PID control and the system response represent a polynomial with hysteresis. The experimentation setup was determined. A set of experimentations were delimited in order to extract from the laboratory BBW system the most relevant information e.g., the rise time, current vs. position relationship, and pressure vs. position relationship.

Experiments' considerations:

(a) A position sweep is necessary to verify the longitudes of the dead zone and the operative zone

(b) If the position of the dead zone and the active zone is the same, all the experiments will start in the dead zone (from constant current (4 amperes)). This experiment aims to graph the dead zone and the operative zone for the master piston of the system.

The master piston has 2 main states, dead zone and operative zone, and a characterization map was realized in order to obtain a direct relation between the piston position and the pressure generated into the calipers. The setpoint signal for this experiment will be a step signal with 4 amperes of magnitude that will be fed to the system in order to obtain the position and pressure responses.

The most effective closed loop structure for $\mathrm{BBW}$ is given in Figure 5, where the pressure controller is the feedforward controller and the feedback effect corresponds to position control. At the cost of an additional measure, the piston position proves dynamically favorable for the control of the BBW system.

To analyze the data, the input signals as well as outputs of the 12 experiments are plotted in 5 different graphics: current displacement, current pressure, displacement pressure, time displacement, and time pressure. The graphics are shown in Figure 6; furthermore, two more graphics are obtained by plotting the displacement and pressure against the time as it is shown in Figure 7.

Assume that the BBW system is one linear system, whose transfer function form is given as

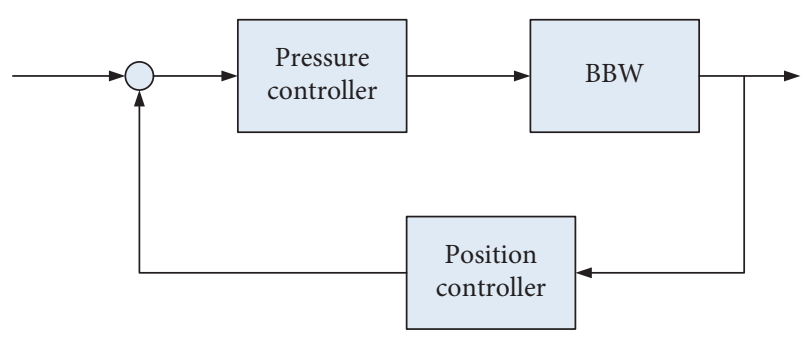

Figure 5: Closed loop structure BBW.

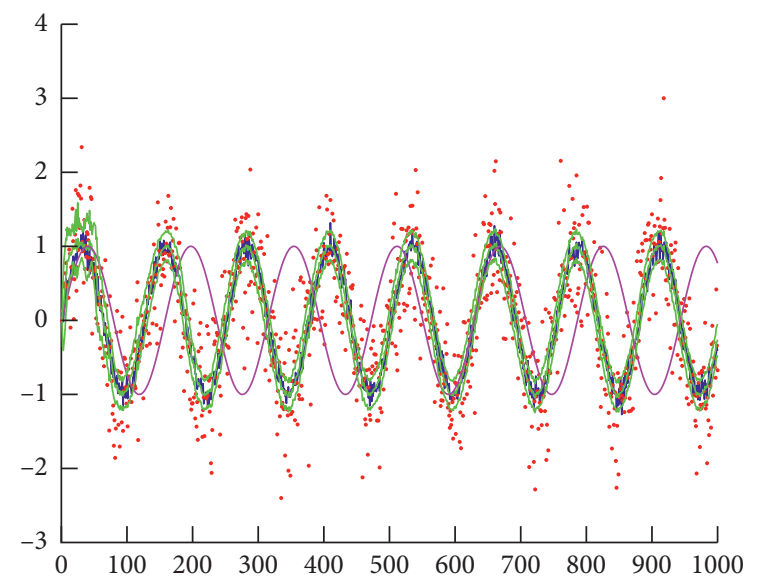

Figure 6: 5 different graphics.

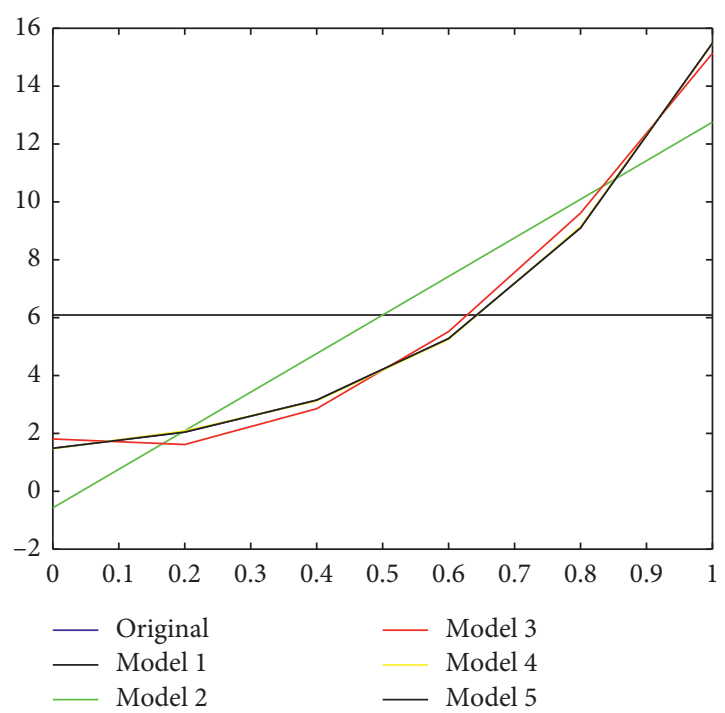

Figure 7: Other two graphics.

$$
P(z)=\frac{(z-1.2)(z-0.4)}{z(z-0.3)(z-0.8)}
$$

The pressure controller in feedforward part $C_{1}(z, \theta)$ is used as

$$
C_{1}(z, \theta)=\alpha^{T}(z) \theta=\left[\frac{z^{2}}{z^{2}-z} \frac{z}{z^{2}-z} \frac{1}{z^{2}-z}\right]\left[\begin{array}{l}
\theta_{1} \\
\theta_{2} \\
\theta_{3}
\end{array}\right]
$$




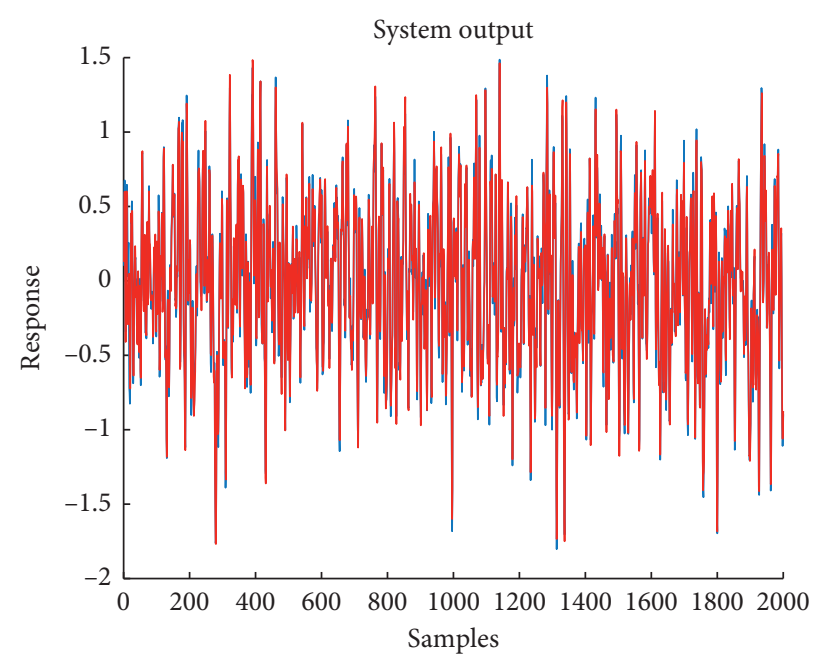

Figure 8: Comparisons of the outputs for the brake-by-wire system.

Its true form is given as

$$
C_{1}(z, \theta)=\alpha^{T}(z) \theta=\left[\frac{z^{2}}{z^{2}-z} \frac{z}{z^{2}-z} \frac{1}{z^{2}-z}\right]\left[\begin{array}{c}
0.86 \\
0.2 \\
0.1
\end{array}\right]
$$

where this true form is unknown, and it is used to analyze the controller performance here.

The expected closed loop transfer function $M(z)$ is chosen as that

$$
M(z)=\frac{z(z-1)\left(0.86 z^{4}-1.1 z^{3}+3.9 z^{2}+0.8 z+0.48\right)}{z^{7}-3 z^{6}-0.96 z^{5}-0.72 z^{4}-0.93 z^{3}+3.9 z^{2}+0.8 z+0.48}
$$

No expected sensitivity function $S(z)$ is considered, i.e., $S(z)=0$. Then, based on equation (18), feedback controller, i.e., position controller, is that

$$
C_{2}(z)=\frac{1-S(Z)}{M(z)}=\frac{1}{M(z)}
$$

Applying those input signals in Figures 6 and 7 in the considered closed loop system and collecting the corresponding output signals, set the number of data points be 2000 , and then, data-driven control and iterative idea are proposed to identify that unknown plant $P(z)$ and design the pressure controller $C_{1}(z)$. The initial parameters, existing in $C_{1}(z)$, are set as follows:

$$
\theta=\left[\begin{array}{lll}
0.75 & 0.25 & 0.15
\end{array}\right]^{T} .
$$

Due to $M(z)$ and $S(z)$ are given in advance, then $C_{2}(z)=$ $((1-S(Z)) / M(z))=(1 / M(z))$ is easily yielded. To show the control performance for designing that pressure control, we compare the closed loop output signals with respect to the true system $\left\{P(z), C_{1}(z), C_{2}(z)\right\}$ and the estimated system $\left\{\widehat{P}(z), \widehat{C}_{1}(z), C_{2}(z)\right\}$, respectively. If these two closed loop output signals are the same or their error is tolerable, then we can regard that the estimated system converges to its true system, i.e., $\widehat{P}(z) \longrightarrow P(z), \widehat{C}_{1}(z) \longrightarrow C_{1}(z)$. Figure 8 shows these two output signals. The red curve is the true closed loop output signals, and the black dots correspond to the estimated closed loop output signals. From Figure 8, we see they correspond to each other, expect for the higher and lower part. Within the interval $[8,16]$, the true outputs coincide with the estimated outputs.

\section{Conclusion}

In this paper, data-driven control is proposed to design two controllers, existing in one closed loop system simultaneously. Firstly, we parametrize controllers by two parameter vectors and then apply the iterative idea to identify them. Secondly, for more general controllers, the iterative idea is also introduced to identify the unknown plant and design controllers. Thirdly, the output-input sensitivity functions are defined for three kinds of disturbances; then, our derived results show that some equities do not vary with the change of the plant. Stability analysis based on the output-input sensitivity functions is our next work.

\section{Data Availability}

The data used to support the findings of this study are available from the corresponding author upon request. 


\section{Disclosure}

This paper was not presented at any IFAC meeting.

\section{Conflicts of Interest}

The authors declare that there are no conflicts of interest regarding the publication of this paper.

\section{Acknowledgments}

This work was partially supported by Jiangxi Provinical National Science Foundation (no. 20202BAL202020).

\section{References}

[1] G. Baggio, V. Katewa, and F. Pasqualetti, "Data driven minimum energy controls for linear systems," IEEE Control Systems Letters, vol. 3, no. 3, pp. 589-594, 2019.

[2] C. De Persis and P. Tesi, "Formulas for data driven control: stabilization, optimality and robustness," IEEE Transactions on Automatic Control, vol. 65, no. 3, pp. 909-924, 2020.

[3] Z.-S. Hou and Z. Wang, "From model-based control to datadriven control: survey, classification and perspective," Information Sciences, vol. 235, no. 2, pp. 3-35, 2013.

[4] C. Novara, S. Formentin, and S. M. Savaresi, "Data driven design for two degree of freedom nonlinear controllers," Automatica, vol. 72, no. 2, pp. 19-27, 2016.

[5] M. Campi and E. Weyer, "Finite sample properties of system identification methods," IEEE Transactions on Automatic Control, vol. 47, no. 13, pp. 29-34, 2002.

[6] S. Dean, H. Mania, and N. Matni, "On the sample complexity of the linear quadratic regulator," Foundations of Computational Mathematics, vol. 20, no. 3, pp. 633-679, 2020.

[7] H. J. Van Warrde and J. Eising, "Data informativity: a new perspective on data driven analysis and control," IEEE Transactions on Automatic Control, vol. 99, pp. 4753-4768, 2020.

[8] J. C. Willems, P. Rapisarda, and L. Markovsky, "A note on persistency of excitation," Systems Control Letter, vol. 54, no. 4, pp. 325-329, 2005.

[9] B. Rechat, "A tour of reinforcement learning: the view from continuous control," Annual Review of Control, Robotics and Autonomous Systems, vol. 3, no. 2, pp. 253-279, 2019.

[10] J. Wang, "Zonotope parameter identification for virtual reference feedback tuning control," International Journal of Systems Science, vol. 50, no. 2, pp. 351-364, 2019.

[11] J. Wang, "Finite sample properties of virtual reference feedback tuning with two degrees of freedom controllers," ISA Transactions, vol. 99, no. 6, pp. 37-49, 2020.

[12] J. Wang, Y. Wang, and R. A. Ramirez-Mendoza, "Adaptive iterative correlation tuning for closed loop system with two parametrized controllers," International Journal of Systems Science, vol. 52, no. 9, pp. 1835-1849, 2021.

[13] F. Bianchi and V. Breschi, "Model structure selection for switched NARX system identification: a randomized approach," Automatica, vol. 125, no. 2, Article ID 109415, 2021.

[14] F. Bianchi and M. Prandini, "A randomized two stage iterative method for switched nonlinear system identification," Nonlinear Analysis-Hybrid Systems, vol. 35, no. 4, Article ID 100818, 2020.

[15] D. P. Bertsekas, "Affine monotonic and risk sensitive models in dynamic programming," IEEE Transactions on Automatic Control, vol. 64, no. 8, pp. 3117-3128, 2019. 